\title{
A phase I trial of 1,3-bis(2-chloroethyl)-1- nitrosourea plus temozolomide: A North American Brain Tumor Consortium study ${ }^{1}$
}

\author{
S. Clifford Schold, Jr., ${ }^{2}$ John G. Kuhn, Susan M. Chang, Michael E. Bosik, \\ H. Ian Robins, Manesh P. Mehta, Alexander M. Spence, Dorcas Fulton, \\ Karen L. Fink, and Michael D. Prados
}

University of Texas Southwestern Medical Center, Dallas, TX 75214 (S. C.S., K.L.F.); University of Texas Health Science Center at San Antonio, San Antonio, TX 78284 (J.G.K.); University of California School of Medicine, San Francisco, CA 94143 (S.M.C., M.D.P.); University of Pittsburgh, Pittsburgh, PA 15213 (M.E.B.); University of Wisconsin, Madison, WI 53792 (H.I.R., M.P.M.); University of Washington, Seattle, WA 98195 (A.M.S.); aand Cross Cancer Institute, Alberta, T6B $1 Z 2$ Canada (D.F.)

The North American Brain Tumor Consortium conducted a phase I trial of the combination 1,3-bis(2chloroethyl)-1-nitrosourea (BCNU) and temozolomide. Eligibility included a patient with a cancer type that was considered refractory to standard therapy. Prior nitrosourea treatments were not permitted. There were parallel dose escalations in two treatment schedules. Forty-five patients were enrolled during an 18-month period. The maximum tolerated doses (MTDs) when temozolomide followed BCNU (Arm A) were temozolomide at $550 \mathrm{mg} / \mathrm{m}^{2} /$ p.o. and $\mathrm{BCNU}$ at $150 \mathrm{mg} / \mathrm{m}^{2} /$ i.v.), whereas the MTD when temozolomide preceded BCNU (Arm B) was temozolomide at $400 \mathrm{mg} / \mathrm{m}^{2} / \mathrm{p} .0$. and BCNU at $100 \mathrm{mg} / \mathrm{m}^{2} /$ i.v. Toxicity was predominantly

Received 22 June 1999, accepted 12 July 1999.

${ }^{1}$ This study was supported by grants CA-62399, CA-62426, CA-54174, CA-62428, and CA-62421 from the National Cancer Institute.

${ }^{2}$ Address correspondence and reprint requests to S. Clifford Schold, Jr., M.D., Duke University Medical Center, Room 201B Bryan Research Building, P.O. Box 2900, Durham, NC 27710.

${ }^{3}$ Abbreviations used are as follows: AGT, $O^{6}$-alkylguanine-DNA alkyltransferase; AUC, area under the curve; BCNU, 1,3-bis(2-chloroethyl)1-nitrosourea; MTD, maximum tolerated dose. hematologic, although there were three instances of pulmonary toxicity, which in one case could have represented potentiation of nitrosourea-induced pulmonary fibrosis. The half-life of temozolomide was $1.86( \pm 0.31)$ h. There was a moderate relationship between dose and peak concentration and a strong relationship between dose and plasma concentration time curve. Pharmacokinetic parameters of temozolomide were unaffected by the treatment schedule, so the difference in MTD between the schedules is likely due to a biologic rather than a pharmacokinetic sequence interaction. There were 9 partial responses among 43 patients evaluable for response, including 5 of 25 with a histologic diagnosis of glioblastoma. The recommended dose and schedule for phase II trials of this regimen are BCNU $150 \mathrm{mg} / \mathrm{m}^{2} /$ i.v. followed in $2 \mathrm{~h}$ by temozolomide $550 \mathrm{mg} / \mathrm{m}^{2} /$ p.o. repeated every 6 weeks. We are also recommending screening and periodic pulmonary function testing during treatment to assess the possible potentiation of nitrosourea-induced pulmonary fibrosis. Neuro-Oncology 2, 34-39, 2000 (Posted to Neuro-Oncology [serial online], Doc. 99-26, December 9, 1999. URL <neuro-oncology.mc.duke.edu>)

$\mathrm{T}$ Temozolomide is an orally administered antineoplastic agent that acts by forming a methyl adduct in tumor DNA. It has shown promising activity in early phase clinical trials, particularly against CNS tumors (Newlands et al., 1992, 1997). Since BCNU ${ }^{3}$ (and other nitrosoureas) also has activity against brain 
tumors, the combination of temozolomide and BCNU were evaluated preclinically. These experimental results indicated therapeutic synergy, as well as schedule-dependent enhanced toxicity (Plowman et al., 1994). The North American Brain Tumor Consortium conducted a phase I evaluation of the combination of these two agents and incorporated into the design a randomized assignment to one of two drug administration schedules (BCNU followed by temozolomide, Arm A, or temozolomide followed by BCNU, Arm B). The results from this phase I trial, with recommendations about the appropriate doses and other caveats for subsequent trials, are presented in this paper.

\section{Materials and Methods}

\section{Eligibility}

Eligibility requirements for this study included a histologic diagnosis of cancer (excluding leukemia) that was considered refractory to standard therapy. Prior radiotherapy must have been completed at least 6 weeks before registration, and prior chemotherapy must have been administered at least 4 weeks before registration. All toxicities from prior treatment must have resolved. No more than one prior chemotherapy regimen was permitted, and no prior treatment with nitrosoureas was permitted. At registration, patients must have had a performance status of 0-2 by Southwest Oncology Group criteria, adequate bone marrow reserve (absolute neutrophil count $>1799 /$ liter, platelets $>124,000 /$ liter, and hemoglobin $>8.9 \mathrm{~g} \%$ ), a serum creatinine of $<1.5 \mathrm{mg} / \mathrm{dl}$ (or a creatinine clearance of $>70 \mathrm{ml} / \mathrm{min}$ ), a serum bilirubin of $<1.5 \mathrm{mg} / \mathrm{dl}$, and a serum SGOT of $<2$ times the institutional upper limit of normal. All blood studies pertaining to eligibility must have been done within 14 days of registration. In addition, patients with other medical illnesses that, in the opinion of the investigator, would compromise the patient's ability to tolerate the therapy and patients who were pregnant or nursing were excluded. Finally, there must have been evidence of evaluable or measurable disease at the time of registration. Patients with primary or metastatic CNS tumors must have had a baseline imaging study (enhanced CT or MRI) performed within 21 days of registration while the patient was taking a stable dose of corticosteroids.

\section{Treatment Schedule and Randomization}

Immediately after registration, patients were randomly assigned to one of two treatment schedules. If assigned to Arm A, patients received BCNU first, followed $2 \mathrm{~h}$ later by temozolomide. If assigned to Arm B, patients received temozolomide first, followed $4 \mathrm{~h}$ later by BCNU. Patients were randomly assigned to Arm A or Arm B as long as accrual was open on both arms of the study at a given dose level. If a sufficient number of patients had been enrolled at a given dose level, all patients were assigned to the other dose level until full accrual at that dose level had been achieved. Treatment was given within 3 days of registration and randomization.

\section{Medication Dosage}

Commercially available BCNU was given by i.v. infusion over $1 \mathrm{~h}$. Temozolomide was obtained from the National Cancer Institute and was given orally. Both drugs were given on day 1 of a 42-day cycle. Five dose levels were evaluated in the trial. The doses (in $\mathrm{mg} / \mathrm{m}^{2}$ ) were as follows: level 1, BCNU 50 and temozolomide 175; level 2, BCNU 75 and temozolomide 275; level 3, BCNU 100 and temozolomide 400; level 4, BCNU 150 and temozolomide 550; level 5, BCNU 200 and temozolomide 750.

\section{Determination of the MTD}

A minimum of 3 patients were treated on each dose level and schedule. If 1 of 3 experienced dose-limiting toxicity, an additional three patients were treated on that dose level and schedule. The MTD was defined as the maximum dose at which 0 of 3 or 1 of 6 patients experienced dose-limiting toxicity and above which 2 or more patients experienced dose-limiting toxicity. An MTD was determined independently for each treatment schedule. Once the MTD was determined, an additional 6 patients were treated on that dose level and schedule to further define toxicity of the regimen.

Dose-limiting toxicity was defined as grade 3 or 4 nonhematologic toxicity (excluding nausea and vomiting), grade 4 nausea and vomiting, grade 4 thrombocytopenia $(<25,000 /$ liter $)$ for 5 days, grade 4 neutropenia $(<500 /$ liter) for 7 days, or grade 4 neutropenia of any duration with fever requiring hospital admission. The Southwest Oncology Group toxicity criteria were used.

\section{Toxicity Evaluation}

All patients were observed for $2.5 \mathrm{~h}$ after initial administration of temozolomide. Complete blood counts were performed at least weekly. Routine blood chemistry studies were performed at least every 6 weeks prior to each cycle of therapy.

\section{Dosage Adjustment}

One dose reduction was permitted per individual patient if unacceptable toxicity occurred. If severe toxicity again occurred at the lower dosage, the patient was removed from the study. Dose escalations in individual patients were permitted only if the patient had experienced no dose-limiting toxicity and a minimum of 3 patients had been treated at the higher level on the same dosing schedule and had experienced no dose-limiting toxicity.

\section{Response Evaluation}

Imaging studies (usually contrast-enhanced MRIs) were performed after every alternate cycle of therapy (approximately every 12 weeks) and as clinically indicated. Because this was a phase I study, no formal response data were required. However, if measurable tumor was present, the patient was declared to have had a complete response if all evidence of tumor disappeared, a partial response if there was a $50 \%$ or greater decrease in the 
product of the perpendicular diameters of the tumor, and progressive disease if the tumor enlarged by $25 \%$ or more. All other patients were considered to have stable disease. The response category was assigned based on the patient's best response, no matter what the duration of the response. If the dose of corticosteroids was higher at the evaluation point than at baseline, complete response and partial response could not be declared. These patients were considered to have either stable or progressive disease.

\section{Sample Collection, Preparation, and Analysis}

Pharmacokinetic studies were performed during the patient's first treatment cycle. Blood samples $(5 \mathrm{ml})$ were collected in prechilled, heparinized (nonseparator) tubes prior to temozolomide administration and at the following times after oral administration: 10, 20, 30, 60, and 90 min and 2, 4, 6, 8 and $24 \mathrm{~h}$. The blood was separated by centrifugation $\left(4^{\circ} \mathrm{C}\right)$ for $10 \mathrm{~min}$. A $1-\mathrm{ml}$ aliquot of plasma was transferred to screwtop polyethylene tubes containing $0.1 \mathrm{ml}$ of $1.0 \mathrm{~N} \mathrm{HCl}$ and stored at $-20^{\circ} \mathrm{C}$ or lower until high pressure liquid chromatography analysis.

A modified high pressure liquid chromatography procedure previously reported by Newlands et al. (1992) was used to determine the plasma concentrations of temozolomide. Analytical standards of temozolomide and the internal standard, ethazalastone, were obtained from the Drug Synthesis and Chemistry Branch, Developmental Therapeutics Program, at the National Cancer Institute. Briefly, a sample of $500 \mu \mathrm{l}$ of acidified plasma containing $20 \mu \mathrm{l}$ (400 ng) of internal standard was double-extracted with ethylacetate and evaporated to dryness under gentle nitrogen. The residue was redissolved with $300 \mu \mathrm{l}$ of mobile phase (acetonitrile/0.1\% acetic acid), vortexed, and filtered, and $75 \mu \mathrm{l}$ was injected via an autosampler into a high pressure liquid chromatography system. The system consisted of a Waters Sperisorb S5 ODS2 column (4.63250 mm; Milford, Mass.) preceded by a Novapak C18 precolumn (Waters) with the mobile phase pumped at a flow rate of $1 \mathrm{ml} / \mathrm{min}$ and ultraviolet detection set at $316 \mathrm{~nm}$. The assay was linear over the range of $0.02-50 \mu \mathrm{g} / \mathrm{ml}$.

The pharmacokinetic parameters for temozolomide were calculated using model-independent methods (Gibaldi, 1984). Peak plasma concentrations $\left(\mathrm{CP}_{\max }\right)$ and the time at which they occurred $\left(\mathrm{T}_{\max }\right)$ were determined by inspection of the individual patient's concentrationtime data. Elimination rate constants were estimated by linear regression of the last two-three data points on the terminal log linear portion of the concentration-time curves. Terminal half-lives $\left(\mathrm{T}_{1 / 2}\right)$ were calculated by dividing 0.693 by the terminal elimination rate constant. The AUC was determined by the trapezoidal method up to the last datum point and extrapolated to infinity. Clearance $(\mathrm{CL} / \mathrm{F})$ was estimated by dividing the dose $\left(\mathrm{mg} / \mathrm{m}^{2}\right)$ of temozolomide by the AUC.

Differences in the pharmacokinetic parameters between the two dosing schedules were assessed using a paired $t$ test with a $P<0.05$ as the a priori level of significance.

\section{Ethical Considerations and Adverse Event Reporting}

The protocol was approved by the Institutional Review Board of each participating institution. All adverse events were reported to the study coordinator by telephone within $24 \mathrm{~h}$. Supporting documentation was then submitted to the National Cancer Institute and the individual Institutional Review Board within 10 days.

\section{Results}

There were 45 patients enrolled on this protocol during an 18 -month period. Of these, 42 were considered eligible for toxicity evaluation. Two patients did not complete the first cycle and died with progressive disease before 6 full weeks of toxicity data could be accumulated. One patient was inadvertently given a second cycle of therapy beginning before the end of the first cycle. This patient went on to receive 4 cycles of therapy and is considered to be evaluable for response but not for toxicity because the assessment of toxicity was based solely on the first full cycle of therapy. Thus, 43 patients were eligible for response.

As indicated in Table 1, the numbers of patients treated on each dose level and schedule (arm) were 4 on $1 \mathrm{~A}, 3$ on $1 \mathrm{~B}, 3$ on $2 \mathrm{~A}, 3$ on $2 \mathrm{~B}, 3$ on $3 \mathrm{~A}, 10$ on $3 \mathrm{~B}, 12$ on $4 \mathrm{~A}, 3$ on $4 \mathrm{~B}$, and 4 on $5 \mathrm{~A}$. No patient experienced doselimiting toxicity on the $1 \mathrm{~A}, 1 \mathrm{~B}, 2 \mathrm{~A}, 2 \mathrm{~B}$, and $3 \mathrm{~A}$ dose schedules (arms). Of the 12 patients treated at the $4 \mathrm{~A}$ dose schedule, 2 experienced dose-limiting toxicity. All three evaluable patients treated at the $5 \mathrm{~A}$ level had doselimiting toxicity. Consequently, the MTD on the Arm A schedule was dose level 4 . Of the 9 patients evaluable for toxicity treated on the $3 \mathrm{~B}$ schedule, 2 experienced doselimiting toxicity. Two of 3 patients treated at the 4B level had dose-limiting toxicity. Consequently, the MTD on the Arm B schedule was dose level 3.

Table 1. Phase I BCNU plus temozolomide: Accrual and dose-limiting toxicity

\begin{tabular}{lcccc}
$\begin{array}{c}\text { Dose } \\
\text { level }\end{array}$ & Arm $^{\mathrm{a}}$ & $\begin{array}{c}\text { Number of } \\
\text { patients } \\
\text { treated }\end{array}$ & $\begin{array}{c}\text { Number } \\
\text { evaluable } \\
\text { for toxicity }\end{array}$ & $\begin{array}{c}\text { Number of } \\
\text { patients } \\
\text { with DLT }\end{array}$ \\
\hline 1 & A & 4 & 3 & 0 \\
1 & B & 3 & 3 & 0 \\
2 & A & 3 & 3 & 0 \\
2 & B & 3 & 3 & 0 \\
3 & A & 3 & 3 & 0 \\
3 & B & 10 & 9 & 2 \\
4 & A & 12 & 12 & 2 \\
4 & B & 3 & 3 & 2 \\
5 & A & 4 & 3 & 3 \\
Total & & 45 & 42 & \\
\hline
\end{tabular}

Abbreviations: BCNU, 1,3-bis(2-chloroethyl)-1-nitrosourea; DLT, dose-limiting toxicity.

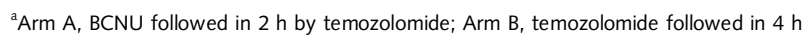
by BCNU.

${ }^{b}$ DLT in first cycle only. 
Nonhematologic toxicity was never greater than grade 2 in the first cycle at any dose level and schedule. The hematologic toxicity was leukopenia, thrombocytopenia, or both. In subsequent cycles, there were 3 instances of grade 4 pulmonary toxicity. Two patients with pulmonary toxicity were treated at the 4B dose level and schedule, and both had primary lung tumors with brain metastases. In both cases, the dose for the second cycle was reduced to level 3 because of hematologic toxicity in the first cycle. Both patients developed acute pulmonary decompensation that was thought in one instance to represent hemorrhage. Neither patient was autopsied. The third patient with pulmonary toxicity subacutely developed dyspnea at rest during the fourth cycle of treatment. She had initially been treated at the $5 \mathrm{~A}$ dose level and schedule but was reduced to $4 \mathrm{~A}$ after the first cycle because of hematologic toxicity. She was found to have a carbon monoxide diffusion capacity of $32 \%$ of the predicted value. Treatment was stopped, and her symptoms gradually improved. She died of recurrent disease but free of pulmonary symptoms 11 months after being enrolled on the protocol. No other significant nonhematologic toxicity occurred in any patient.

There were 9 partial responses to the therapy (Table 2). Five of these were in patients with glioblastoma, 2 in patients with brain metastases, and 2 in patients with anaplastic astrocytoma. Partial responses occurred at dose levels 1, 3, 4, and 5. Five of the partial responses were treated on study Arm A, and 4 were treated on study Arm B. Ten patients remained alive at the time of this analysis, including 5 with a diagnosis of glioblastoma who were alive $11,13,16,18$, and 30 months after enrollment.

Blood samples for pharmacokinetic analysis were obtained from 32 of the 45 patients. Two patients were not evaluable due to incomplete sample collections. The mean $( \pm \mathrm{SD})$ pharmacokinetic parameters for temozolomide for the two treatment schedules (arms) are summarized in Table 3. Oral temozolomide was rapidly absorbed, reaching peak concentrations an average of 1.3 $( \pm 1.3)$ h (range $0.3-6 \mathrm{~h})$ after administration. The harmonic mean half-life for temozolomide was $1.86( \pm 0.31)$ h. Mean total body clearance for temozolomide across all dose levels was $6.08( \pm 0.72) \mathrm{liter} / \mathrm{h} / \mathrm{m}^{2}$. There was a moderate relationship between dose $\left(\mathrm{mg} / \mathrm{m}^{2}\right)$ and peak concentrations $\left(\mathrm{r}^{2}=0.38, P<0.002\right)$ and a strong correlation between dose and AUCs $\left(\mathrm{r}^{2}=0.91, P<0.002\right)$. No significant differences between the two dosing schedules were seen in the pharmacokinetic parameters $\left(\mathrm{T}_{\max }, \mathrm{T}_{1 / 2}\right.$, AUC, or clearance). There was a definite pharmacodynamic relationship between the duration of exposure (AUC) and grade of neutropenia $\left(\mathrm{r}^{2}=0.62, P<0.002\right)$ and thrombocytopenia $\left(\mathrm{r}^{2}=0.74, P<0.002\right)$.

\section{Discussion}

Temozolomide is a methylating agent that has shown activity as a single agent in phase II trials in patients with gliomas (Newlands et al., 1992, 1997). Experimentally, it has also shown therapeutic synergy in combination with nitrosoureas (Plowman et al., 1994), and these results led to development of the phase I trial of temozolomide plus $\mathrm{BCNU}$ that is reported in this paper.
Table 2. Phase I BCNU plus temozolomide: Response by histology

\begin{tabular}{lcccc} 
& \multicolumn{4}{c}{ Response } \\
\cline { 2 - 5 } Histologic diagnosis & PR & SD & PD & NE \\
Glioblastoma & 5 & 10 & 9 & 1 \\
\hline Metastasis & 2 & 1 & 5 & 1 \\
Anaplastic astrocytoma & 2 & 2 & 2 & 0 \\
Mixed anaplastic glioma & 0 & 1 & 1 & 0 \\
Anaplastic oligodendroglioma & 0 & 1 & 0 & 0 \\
Total & 9 & 15 & 17 & 2 \\
\hline
\end{tabular}

Abbreviations: $B C N U, 1,3$-bis(2-chloroethyl)-1-nitrosourea; PR, partial response; $S D$, stable disease; PD, progressive disease; NE not evaluable.

In the preclinical studies of the temozolomide and BCNU combination, there was schedule-dependency. In a series of experiments using human glioma xenografts in athymic mice, Plowman et al. (1994) demonstrated a synergistic therapeutic effect of the combination of BCNU and temozolomide. Animals receiving temozolomide $2 \mathrm{~h}$ before BCNU were much more likely to die from the treatment than animals treated with identical doses in the reverse sequence. The therapeutic effect of the 2 dosing schedules was similar, although the data suggested possible superiority of the sequence in which BCNU was administered first. Consequently, one of the objectives of this trial was to compare two schedules of the combination, namely temozolomide first versus $\mathrm{BCNU}$ first. The data reported here indicate that there is a schedule-dependency in humans as well, at least in terms of the toxicity of the combination. The MTD on the Arm A schedule (BCNU first) was one dosage level higher than the MTD on the Arm B schedule (temozolomide first). The magnitude of the differential toxicity cannot be assessed from our data because the differences between dosage levels were fairly large. Perhaps refinement of the dosages in the two schedules would give more insight into the degree of difference in toxicity, as well as additional data that would help us understand the mechanism of the schedule dependency. Nevertheless, based on the principle that more drug is better and the suggestion in the preclinical studies that administration of BCNU given first produced greater efficacy, we are recommending the Schedule A (BCNU followed in $2 \mathrm{~h}$ by temozolomide) at the 4 th dose level $\left(150 \mathrm{mg} / \mathrm{m}^{2}\right.$ BCNU and $550 \mathrm{mg} / \mathrm{m}^{2}$ temozolomide) for phase II trials.

The only significant toxicity produced by the combination of BCNU and temozolomide in the first cycle of therapy was hematologic, and the dosage recommendations for future trials are based on these results. However, there is a concern that nonhematologic toxicity might be accentuated by the combination as well. This was not addressed directly in this study. There were three patients who developed some form of significant pulmonary dysfunction during the course of treatment and, of course, pulmonary toxicity is the principal nonhematologic toxicity that occurs with BCNU therapy (Hundley and Lukens, 1979). However, it appears that in 2 of the 3 cases in this series, the pulmonary problems were not the typical diffusion restriction pattern of dysfunction usually seen with 
Table 3. Phase I BCNU plus temozolomide: Pharmacokinetic data for the two dosing schedules

Temozolomide dose $\left(\mathrm{mg} / \mathrm{m}^{2}\right)$

\begin{tabular}{|c|c|c|c|c|c|c|c|c|c|}
\hline \multirow{3}{*}{$\frac{\text { Parameter }}{\text { Arm }^{\mathrm{a}}}$} & \multirow{2}{*}{\multicolumn{2}{|c|}{175}} & \multirow{2}{*}{\multicolumn{2}{|c|}{275}} & \multirow{2}{*}{\multicolumn{2}{|c|}{400}} & \multirow{2}{*}{\multicolumn{2}{|c|}{550}} & \multirow{3}{*}{$\begin{array}{c}750 \\
A\end{array}$} \\
\hline & & & & & & & & & \\
\hline & $A$ & $B$ & $A$ & $\mathrm{~B}$ & $A$ & $\mathrm{~B}$ & $A$ & $B$ & \\
\hline & [3] & [3] & {$[2]$} & [3] & [3] & [4] & {$[5]$} & [3] & [4] \\
\hline \multirow{2}{*}{$\mathrm{CP}_{\max }(\mu \mathrm{g} / \mathrm{ml})$} & 7.67 & 12.91 & 19.60 & 20.67 & 27.00 & 21.93 & 27.32 & 20.03 & 27.34 \\
\hline & (3.59) & $(5.34)$ & $(0.28)$ & $(4.35)$ & $(7.22)$ & $(4.17)$ & $(5.45)$ & $(3.17)$ & (12.03) \\
\hline \multirow[t]{2}{*}{$\mathrm{T}_{\max }(\mathrm{h})$} & 2.2 & 0.6 & 0.5 & 0.6 & 0.8 & 0.8 & 1.0 & 2.7 & 2.4 \\
\hline & $(1.8)$ & $(0.4)$ & $(0)$ & $(0.4)$ & $(0.6)$ & $(0.2)$ & $(0.5)$ & $(1.2)$ & $(2.5)$ \\
\hline \multirow[t]{2}{*}{$T_{1 / 2}(h)^{b}$} & 2.0 & 1.8 & 1.9 & 1.9 & 1.9 & 1.8 & 1.8 & 1.9 & 1.9 \\
\hline & $(0.3)$ & $(0)$ & $(0.3)$ & $(0.1)$ & $(0.1)$ & $(0.2)$ & $(0.1)$ & $(0.1)$ & $(0.9)$ \\
\hline \multirow[t]{2}{*}{$\mathrm{AUC}(\mu \mathrm{g} \times \mathrm{h} / \mathrm{ml})$} & 25.93 & 29.89 & 44.58 & 46.57 & 81.10 & 68.16 & 86.39 & 93.33 & 118.51 \\
\hline & $(2.75)$ & $(3.02)$ & (3.79) & $(2.20)$ & $(11.53)$ & (10.31) & $(4.77)$ & $(5.49)$ & $(17.41)$ \\
\hline \multirow[t]{2}{*}{$\mathrm{CL} / \mathrm{F}\left(\mathrm{l} / \mathrm{h} / \mathrm{m}^{2}\right)$} & 6.80 & 5.89 & 6.19 & 5.91 & 4.99 & 5.96 & 6.38 & 5.91 & 6.43 \\
\hline & $(0.77)$ & $(0.60)$ & $(0.52)$ & $(0.28)$ & $(0.66)$ & $(0.83)$ & $(0.38)$ & $(0.35)$ & $(0.90)$ \\
\hline
\end{tabular}

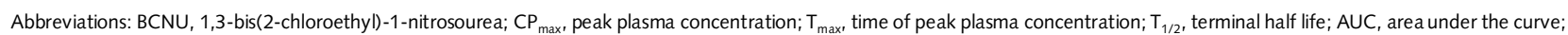
$\mathrm{CL} / \mathrm{F}$, clearance.

Data are means $( \pm S D)$.

${ }^{\mathrm{a}} \mathrm{Arm}$ A schedule, BCNU followed in $2 \mathrm{~h}$ by temozolomide; Arm B schedule, temozolomide followed in $4 \mathrm{~h}$ by BCNU. Numbers in brackets are number of patients.

${ }^{\mathrm{b}}$ Harmonic mean.

BCNU. The third patient with pulmonary toxicity did have a more typical pattern with marked reduction of carbon monoxide diffusion capacity after a total BCNU dose of only $650 \mathrm{mg} / \mathrm{m}^{2}$, a dose considerably lower than that which usually produces pulmonary toxicity when BCNU is given as a single agent. Fortunately, discontinuing therapy resulted in symptomatic improvement in this patient, who experienced a partial response but subsequently died due to disease recurrence 11 months after enrollment. Nevertheless, because we are concerned about chronic pulmonary toxicity in patients treated with this combination, we recommend that pulmonary function tests be included in the eligibility criteria for future trials of this drug combination and that patients with poor baseline pulmonary function be excluded until the effect on pulmonary diffusion capacity can be assessed.

The single-dose pharmacokinetic parameters of temozolomide characterized in this study are in close agreement with the previous study reported by Newlands et al. (1992). The administration sequence of temozolomide (before or after BCNU) had no significant effect on the pharmacokinetic parameters of temozolomide. Therefore, the difference in the MTD observed between the two schedules probably relates to a biological effect rather than a pharmacokinetic sequence interaction. Likewise, the anticonvulsants (phenytoin, phenobarbital, carbamazepine) that many of the patients received concomitantly do not appear to have altered the pharmacokinetic characteristics of temozolomide. This observation is consistent with a drug whose clearance is independent of enzymatic processes (Tsang et al., 1990).

The mechanism of synergy between BCNU and temozolomide is unknown. Temozolomide acts by methylating the $\mathrm{O}^{6}$ position of guanine in DNA (Mitchell and Dolan, 1993). BCNU forms a chloroethyl adduct at the same site. Both the methyl and the chloroethyl adducts are repaired by the DNA repair protein AGT, and it is known that in preclinical systems, high levels of tumor AGT protect against the cytotoxic effects of either BCNU or temozolomide (Friedman et al., 1995; Schold et al., 1989). AGT is inactivated when it reacts with an alkyl adduct, and tumors (as well as normal tissues) can be sensitized to the effects of alkylating drugs, such as BCNU, by pretreatment with compounds that react with AGT, such as $\mathrm{O}^{6}$-benzylguanine (Felker et al., 1993; Friedman et al., 1992). Possibly, the greater toxicity of the dosing schedule in which temozolomide is administered first is due to sensitization of the bone marrow cells to BCNU by temozolomide-induced inhibition of AGT. If that is the mechanism, one could expect a synergistic therapeutic effect as well. However, no direct evidence has shown this as the primary mechanism of the synergy of these two compounds. Clearly, some patients whose tumors have acquired resistance to nitrosoureas retain sensitivity to procarbazine, an agent that produces the same DNA adducts as temozolomide, so there are undoubtedly mechanisms of resistance other than the AGT protein (Friedman et al., 1997). The synergy of BCNU and temozolomide could, therefore, be due to giving, concurrently, agents that have different mechanisms of drug resistance.

Although the primary purpose of this phase I trial was to determine the appropriate dosages of the two agents for subsequent trials, it was encouraging that some responses were seen. Because response was not a primary objective of the trial, we did not require central review of the imaging studies. Consequently, the response data should be viewed critically. Accurate data about the activity of this combination at the MTD will be forthcoming from a current phase II trial of the North American Brain Tumor Consortium (NABTC-9701). This trial seeks to estimate not only the response rate of recurrent glioblastoma to this regimen, but also the response rate of newly diagnosed anaplastic astrocytoma prior to radiotherapy. 


\section{References}

Felker, G.M., Friedman, H.S., Dolan, M.E., Moschel, R.C., and Schold, C. (1993) Treatment of subcutaneous and intracranial brain tumor xenografts with $\mathrm{O}^{6}$-benzylguanine and 1,3-bis(2-chloroethyl)-1nitrosourea. Cancer Chemother. Pharmacol. 32, 471-476.

Friedman, H.S., Dolan, M.E., Moschel, R.C., Pegg, A.E., Felker, G.M., Rich, J., Bigner, D.D., and Schold, S.C., Jr. (1992) Enhancement of nitrosourea activity in medulloblastoma and glioblastoma multiforme. J. Natl. Cancer Inst. 84, 1926-1931.

Friedman, H.S., Dolan, E.E., Pegg, A.E., Marcelli, S., Keir, S., Catino, J.J., Bigner, D.D., and Schold, S.C., Jr. (1995) Activity of temozolomide in the treatment of central nervous system tumor xenografts. Cancer Res. 55 , 2853-2857.

Friedman, H.S., Johnson, S.P., Doug, Q., Schold, S.C., Rasheed, B.K., Bigner, S.H., Ali-Osman, F., Dolan, E., Colvin, O.M., Houghton, P., Germain, G., Drummond, J.T., Keir, S., Marcelli, S., Bigner, D.D., and Modrich, P. (1997) Methylator resistance mediated by mismatch repair deficiency in a glioblastoma multiforme xenograft. Cancer Res. 57, 2933-2936.

Gibaldi, M. (Ed.) (1984) Biopharmaceutics and Clinical Pharmacokinetics. Third edition. Philadelphia, Penn.: Lea \& Febiger.

Hundley, R.F., and Lukens, J.N. (1979) Nitrosourea-associated pulmonary fibrosis. Cancer Treat. Rep. 63, 2128-2130.
Mitchell, R.B., and Dolan, M.E. (1993) Effect of temozolomide and dacarbazine on $\mathrm{O}^{6}$-alkylguanine-DNA alkyltransferase activity and sensitivity of human tumor cells and xenografts to 1,3-bis(2-chloroethyl)-1nitrosourea. Cancer Chemother. Pharmacol. 32, 59-63.

Newlands, E.S., Blackledge, G.R.P., Slack, J.A., Rustin, G.J., Smith, D.B., Stuart, N.S., Quarterman, C.P., Hoffman, R., Stevens, M.F., and Brampton, M.H. (1992) Phase I trial of temozolomide (CCRG 81045: M\&B 39831: NSC 362856). Br. J. Cancer 65, 287-291.

Newlands, E.S., Stevens, M.F.G., Wedge, S.R., Wheelhouse, R.T., and Brock, C. (1997) Temozolomide: A review of its discovery, chemical properties, pre-clinical development and clinical trials. Cancer Treat. Rev. 23, 35-61.

Plowman, J., Waud, W.R., Koutsoukos, A.D., Rubinstein, L.V., Moore, T.D., and Grever, M.R. (1994) Preclinical antitumor activity of temozolomide in mice: Efficacy against human brain tumor xenografts and synergism with 1,3-bis(2-chloroethyl)-1-nitrosourea. Cancer Res. 54, 3793-3799.

Schold, S.C., Jr., Brent, T.P., von Hofe, E., Friedman, H.S., Mitra, S., Bigner, D.D., Swenberg, J.A., and Kleihues, P. (1989) $\mathrm{O}^{6}$-alkylguanine-DNAalkyltransferase and sensitivity to procarbazine in human brain tumor xenografts. J. Neurosurg. 70, 573-577.

Tsang, L.L.H., Farmer, P.B., Gescher, A., and Slack, J.A. (1990) Characterization of urinary metabolites of temozolomide in humans and mice and evaluation of their cytotoxicity. Cancer Chemother. Pharmacol. 26, 429-436. 